\title{
Analytic adjoint solutions for the quasi-one-dimensional Euler equations
}

\author{
By MICHAEL B. GILES AND NILES A. PIERCE $\dagger$ \\ Oxford University Computing Laboratory, Oxford, OX1 3QD, UK
}

(Received 11 June 1998 and in revised form 8 August 2000)

The analytic properties of adjoint solutions are examined for the quasi-onedimensional Euler equations. For shocked flow, the derivation of the adjoint problem reveals that the adjoint variables are continuous with zero gradient at the shock, and that an internal adjoint boundary condition is required at the shock. A Green's function approach is used to derive the analytic adjoint solutions corresponding to supersonic, subsonic, isentropic and shocked transonic flows in a converging-diverging duct of arbitrary shape. This analysis reveals a logarithmic singularity at the sonic throat and confirms the expected properties at the shock.

\section{Introduction}

Adjoint equations arise naturally in the formulation of methods for optimal aerodynamic design. A single adjoint solution provides the linear sensitivities of an objective function, such as lift or drag, to perturbations in the multiple design variables which parameterize the aerodynamic shape. These sensitivities can then be used to drive a gradient-based optimization procedure.

To outline the approach, we start with a system of nonlinear partial differential equations (e.g. the Euler equations or compressible Navier-Stokes equations) describing a steady flow within some given computational domain. When calculating the two-dimensional flow around an aerofoil, one technique is to use curvilinear coordinates $(\xi, \eta)$ in which the aerofoil surface corresponds to $\eta=0$ (Jameson 1995). Using these coordinates, the p.d.e. can be written as

$$
R(U)=0,
$$

where $U$ is the flow solution and $R$ is a nonlinear differential operator which depends on the mapping from $(\xi, \eta)$ to the Cartesian coordinates $(x, y)$, which in turn depends on the geometry of the aerofoil. Perturbing the geometry changes the mapping, and hence $R$. Linearizing the operator $R(U)$ then leads to the linear p.d.e.

$$
L u=f,
$$

where $f$ is due to the change in the mapping, and $u$ is the resultant linear perturbation to the flow field.

In design optimization, there is interest in the consequential change to some objective function which is to be minimized. Usually, this objective involves an integral over the boundary of the domain, as in the case of drag minimization. However, to

$\dagger$ Present address: Applied Mathematics, California Institute of Technology, Pasadena, CA, 91125, USA. 
simplify this exposition, we will take the objective function $J(U)$ to be an integral over the whole domain $\Omega$, whose linear perturbation $I(u)$ can then be written as an inner product over the domain,

$$
I(u)=(g, u),
$$

for some given function $g(\xi, \eta)$.

Using a direct approach to design, $I(u)$ is determined separately for each design variable by defining the appropriate geometric perturbation $f$ and solving equation (1.2) for $u$. In the adjoint approach, the perturbed functional is evaluated without explicitly calculating the perturbed flow field $u$. This is achieved by using an augmented functional

$$
I=(g, u)-(v, L u-f),
$$

in which the continuous Lagrange multipliers $v$ have been introduced to enforce the constraint that $u$ must satisfy equation (1.2). The adjoint linear operator $L^{*}$ is defined by the identity

$$
(v, L u)=\left(L^{*} v, u\right),
$$

for all $u, v$ satisfying the appropriate homogeneous boundary conditions. Using this identity,

$$
I=(v, f)-\left(L^{*} v-g, u\right)=(v, f)
$$

is obtained, provided $v$ is the solution of the adjoint equation

$$
L^{*} v=g .
$$

The adjoint approach provides exactly the same final answer as the direct linear perturbation analysis. The benefit of the adjoint approach is that the computational cost can be significantly lower. If there are $N$ design variables, then a direct approach requires $N$ solutions of equation (1.2), each with a different function $f$, to obtain the linear flow perturbations $u$. On the other hand, with the adjoint approach, equation (1.3) has to be solved only once for the function $g$ corresponding to the objective function of interest. Since solving equations (1.2) and (1.3) requires roughly equal computational effort, the overall savings become substantial as the number of design variables increases.

In the last ten years, considerable effort has been devoted to the development of optimal design methods based on the adjoint approach. Some methods use curvilinear coordinates and the differential adjoint, as outlined above (see e.g. Jameson 1988, 1995, 1999; Reuther et al. 1996, 1999a, b; Jameson, Pierce \& Martinelli 1998). Other methods first discretize the nonlinear p.d.e. and then use the adjoint (transpose) of the linear discrete matrix operator (Elliott \& Peraire 1997; Anderson \& Bonhaus 1999). For a more comprehensive introduction to adjoint methods in aerodynamic design and a discussion of the relative advantages of the two main approaches, see Giles \& Pierce (2001). For a review of the latest developments in design optimization using adjoint equations, see Newman et al. (1999).

Recently, adjoint solutions have been recognized as providing a means of computing and minimizing errors in fluid dynamics simulations, and, in particular, the errors in integral outputs such as lift and drag. Suppose $U_{h}$ is an approximate numerical solution of equation (1.1). Defining $u$ to be the numerical error (the difference between the numerical and analytic solutions) gives

$$
R\left(U_{h}-u\right)=0 .
$$


Linearization about the numerical solution then yields

$$
L u=f, \quad f \equiv R\left(U_{h}\right) .
$$

Defining the adjoint solution in the same way as before, the leading-order error in the integral objective function is given by

$$
(g, u)=(v, f)=\left(v, R\left(U_{h}\right)\right) .
$$

This result can be used in grid adaptation, for example by refining any cell in which an estimate of the local product $v^{T} R\left(U_{h}\right)$ multiplied by the cell area exceeds some threshold, to try to achieve the maximum reduction in the magnitude of the error for a given computational effort (Johnson, Rannacher \& Boman 1995; Paraschivoiu, Peraire \& Patera 1997; Becker \& Rannacher 1998; Süli 1998). Alternatively, this error term can be carefully evaluated and used to correct the value of the objective function given by the calculated flow field. For the two-dimensional Poisson equation and the quasi-one-dimensional Euler equations, this has been shown to lead to corrected values of twice the order of accuracy of the flow-field solution (Giles \& Pierce 1998, 1999; Pierce \& Giles 1998, 2000).

While significant effort has been dedicated to developing methods for calculating adjoint solutions to compressible flow equations, there has been little discussion of the properties of the adjoint solutions themselves (see Giles \& Pierce 1997, 1998). The present work investigates the analytic properties of adjoint solutions for the quasione-dimensional Euler equations. The standard formulation of the adjoint equations using Lagrange multipliers (Jameson 1995) is extended to include the analysis of a shock. Explicit enforcement of the steady Rankine-Hugoniot conditions through an additional Lagrange multiplier leads to the result that at the shock, the adjoint variables are continuous and there is an internal adjoint boundary condition. This is consistent with a characteristic viewpoint which indicates that one internal adjoint boundary condition is needed owing to the disparity in the number of adjoint characteristics entering and leaving the shock. However, the conclusions differ from those of previous investigators (see Iollo, Salas \& Ta'asan 1993; Iollo \& Salas 1996; Cliff, Heinkenschloss \& Shenoy 1996, 1998).

The analytic adjoint solutions are then derived in closed form for all Mach regimes. This is accomplished by constructing the Green's functions for the linearized Euler equations, including the linearized Rankine-Hugoniot conditions, using an extension of the approach developed by Giles \& Pierce (1997) for shock-free quasi-onedimensional flows. These solutions confirm the expected behaviour at the shock and reveal a logarithmic singularity in the adjoint variables at the sonic point. These insights are helpful in understanding the requirements for developing effective numerical methods (Giles \& Pierce 1998). In this regard, it is hoped that the analytic solutions will also serve as a useful set of test cases for researchers developing adjoint numerical methods.

\section{Adjoint problem formulation}

The quasi-one-dimensional Euler equations for steady flow in a duct of cross-section $h(x)$, on the interval $-1 \leqslant x \leqslant 1$, may be written as

$$
R(U, h) \equiv \frac{\mathrm{d}}{\mathrm{d} x}(h F)-\frac{\mathrm{d} h}{\mathrm{~d} x} P=0,
$$


where

$$
U=\left(\begin{array}{c}
\rho \\
\rho q \\
\rho E
\end{array}\right), \quad F=\left(\begin{array}{c}
\rho q \\
\rho q^{2}+p \\
\rho q H
\end{array}\right), \quad P=\left(\begin{array}{l}
0 \\
p \\
0
\end{array}\right) .
$$

Here, $\rho$ is the density, $q$ is the velocity, $p$ is the pressure, $E$ is the total energy and $H$ is the stagnation enthalpy. The system is closed by the equation of state for an ideal gas

$$
H=E+\frac{p}{\rho}=\frac{\gamma}{\gamma-1} \frac{p}{\rho}+\frac{1}{2} q^{2} .
$$

If the solution contains a shock at $x_{s}$, the Rankine-Hugoniot jump condition

$$
[F]_{x_{s}^{-}}^{x_{s}^{+}}=0
$$

connects the smooth solutions on either side.

For design applications, linearization of $R$ with respect to perturbations in the flow solution, $u$, and the geometry, $\tilde{h}$, produces

$$
L u-f \equiv\left(\frac{\mathrm{d}}{\mathrm{d} x}(h A u)-\frac{\mathrm{d} h}{\mathrm{~d} x} B u\right)-\left(\frac{\mathrm{d} \tilde{h}}{\mathrm{~d} x} P-\frac{\mathrm{d}}{\mathrm{d} x}(\tilde{h} F)\right)=0,
$$

where $A=(\partial F / \partial U)$ and $B=(\partial P / \partial U)$.

We choose the objective function to be the integral of pressure along the duct,

$$
J=\int_{-1}^{1} p \mathrm{~d} x=\int_{-1}^{x_{s}} p \mathrm{~d} x+\int_{x_{s}}^{1} p \mathrm{~d} x
$$

since this mimics the lift integral which is of importance in aeronautical applications. Other objective functions could also be considered with only minor changes to the analysis to be presented. The perturbation to this 'lift' integral owing to changes in the flow is

$$
I=\int_{-1}^{x_{s}} g^{T} u \mathrm{~d} x+\int_{x_{s}}^{1} g^{T} u \mathrm{~d} x-[p]_{x_{s}^{-}}^{x_{s}^{+}} \delta,
$$

where $g=(\partial p / \partial U)^{T}$, and the third term includes the effect of a linearized displacement $\delta$ in the shock location.

Using continuous Lagrange multipliers $v$ to enforce the differential flow constraints on either side of the shock, and a Lagrange multiplier $v_{s}$ to enforce the RankineHugoniot conditions at the shock, the augmented nonlinear objective function is

$$
J=\int_{-1}^{x_{s}} p \mathrm{~d} x+\int_{x_{s}}^{1} p \mathrm{~d} x-\int_{-1}^{x_{s}^{-}} v^{T} R \mathrm{~d} x-\int_{x_{s}^{+}}^{1} v^{T} R \mathrm{~d} x-h_{s} v_{s}^{T}[F]_{x_{s}^{-}}^{x_{s}^{+}},
$$

where $h_{s} \equiv h\left(x_{s}\right)$. Linearizing this with respect to perturbations in the geometry $\tilde{h}$, the shock location $\delta$ and the flow solution $u$ gives

$$
\begin{aligned}
I= & \int_{-1}^{x_{s}} g^{T} u \mathrm{~d} x+\int_{x_{s}}^{1} g^{T} u \mathrm{~d} x-[p]_{x_{s}^{-}}^{x_{s}^{+}} \delta \\
& -\int_{-1}^{x_{s}^{-}} v^{T}(L u-f) \mathrm{d} x-\int_{x_{s}^{+}}^{1} v^{T}(L u-f) \mathrm{d} x \\
& -h_{s} v_{s}^{T}[A u]_{x_{s}^{-}}^{x_{s}^{+}}-h_{s} v_{s}^{T}\left[\frac{\mathrm{d} F}{\mathrm{~d} x}\right]_{x_{s}^{-}}^{x_{s}^{+}} \delta .
\end{aligned}
$$


After integration by parts and rearrangement, this yields

$$
\begin{aligned}
I= & \int_{-1}^{x_{s}} v^{T} f \mathrm{~d} x+\int_{x_{s}}^{1} v^{T} f \mathrm{~d} x \\
& -\int_{-1}^{x_{s}^{-}}\left(L^{*} v-g\right)^{T} u \mathrm{~d} x-\int_{x_{s}^{+}}^{1}\left(L^{*} v-g\right)^{T} u \mathrm{~d} x \\
& -\delta\left(h_{s} v_{s}^{T}\left[\frac{\mathrm{d} F}{\mathrm{~d} x}\right]_{x_{s}^{-}}^{x_{s}^{+}}+[p]_{x_{s}^{-}}^{x_{s}^{+}}\right) \\
& -\left.h_{s}\left(v_{s}-v\left(x_{s}^{+}\right)\right)^{T} A u\right|_{x_{s}^{+}}+\left.h_{s}\left(v_{s}-v\left(x_{s}^{-}\right)\right)^{T} A u\right|_{x_{s}^{-}} \\
& -\left[h v^{T} A u\right]_{-1}^{1},
\end{aligned}
$$

where the adjoint operator $L^{*}$ is defined by

$$
L^{*} v \equiv-h A^{T} \frac{\mathrm{d} v}{\mathrm{~d} x}-\frac{\mathrm{d} h}{\mathrm{~d} x} B^{T} v .
$$

The idea of the adjoint approach is to define the adjoint problem so as to eliminate the explicit dependence of $I$ on $u$ and $\delta$, giving the adjoint form of the objective function

$$
I=\int_{-1}^{x_{s}} v^{T} f \mathrm{~d} x+\int_{x_{s}}^{1} v^{T} f \mathrm{~d} x=\int_{-1}^{1} v^{T} f \mathrm{~d} x .
$$

To eliminate the dependence on $u, v$ must satisfy the adjoint o.d.e.

$$
L^{*} v-g=0,
$$

and at the shock, $v$ and $v_{s}$ must satisfy

$$
v\left(x_{s}^{-}\right)=v_{s}=v\left(x_{s}^{+}\right),
$$

proving that the adjoint variables are continuous across the shock. Removing the dependence of $I$ on $\delta$ then requires that

$$
h_{s} v^{T}\left(x_{s}\right)\left[\frac{\mathrm{d} F}{\mathrm{~d} x}\right]_{x_{s}^{-}}^{x_{s}^{+}}=-[p]_{x_{s}^{-}}^{x_{s}^{+}},
$$

which is an internal boundary condition at the shock. Noting that

$$
\left[\frac{\mathrm{d} F}{\mathrm{~d} x}\right]_{x_{s}^{-}}^{x_{s}^{+}}=\left[\frac{1}{h} \frac{\mathrm{d} h}{\mathrm{~d} x} P\right]_{x_{s}^{-}}^{x_{s}^{+}},
$$

this reduces to the simple boundary condition

$$
v_{2}\left(x_{s}\right)=-\left(\frac{\mathrm{d} h}{\mathrm{~d} x}\left(x_{s}\right)\right)^{-1} .
$$

Finally, the inlet and exit boundary conditions for the adjoint problem are defined so as to remove the explicit dependence of

$$
\left[h v^{T} A u\right]_{-1}^{1}
$$

on $u$. At a boundary where the flow equations have $n$ incoming characteristics, and hence $n$ imposed boundary conditions, the adjoint equations will thus have 
$(3-n)$ boundary conditions corresponding to an equal number of incoming adjoint characteristics (Giles \& Pierce 1997).

The need for an adjoint boundary condition at the shock can be understood by considering the characteristics of the hyperbolic system. For the adjoint problem, information travels along characteristics in the opposite direction as for the flow problem. Thus, at the shock, there are three outgoing characteristics on the upstream side and one outgoing characteristic on the downstream side. Continuity of the adjoint variables across the shock provides three conditions and the additional shock boundary condition provides a fourth, ensuring that all outgoing characteristics are fully determined.

In Iollo et al. (1993), it is suggested that $v=0$ could be imposed at the shock, but this over-constrains the adjoint problem, in addition to contradicting (2.5). Cliff et al. $(1996,1998)$ conclude that there is a 'shock' in the adjoint variables at the shock location, having proved that the adjoint variables undergo a change of sign across the shock. However, as this change of sign is entirely due to the non-standard coordinate system they employ in formulating the augmented Lagrange, the conclusion that the adjoint variables are discontinuous at the shock is misleading.

A final observation is that the adjoint equation (2.4) and the adjoint shock boundary condition (2.5) together cause the gradient of the adjoint variables to vanish at the shock. This may be seen by writing (2.4) using Jacobians based on the nonconservative flow variables $U_{p}=(\rho, q, p)^{T}$, so that the adjoint equation becomes,

$$
h\left(\begin{array}{ccc}
q & q^{2} & \frac{1}{2} q^{3} \\
\rho & 2 \rho q & \frac{\gamma}{\gamma-1} p+\frac{3}{2} \rho q^{2} \\
0 & 1 & \frac{\gamma}{\gamma-1} q
\end{array}\right) \frac{\mathrm{d} v}{\mathrm{~d} x}=-\left(\begin{array}{c}
0 \\
0 \\
1+\frac{\mathrm{d} h}{\mathrm{~d} x} v_{2}
\end{array}\right),
$$

and the adjoint shock boundary condition produces $(\mathrm{d} v / \mathrm{d} x)=0$ at the shock. This feature is important in understanding the success of certain numerical discretizations in producing the correct adjoint behaviour at the shock, without explicit enforcement of the internal adjoint boundary condition (Giles \& Pierce 1998).

\section{Green's function approach}

To verify the properties of the adjoint solutions and to provide a reference for comparison with numerical results, the analytic adjoint solutions are now derived for both isentropic and shocked transonic flows.

The derivation uses a Green's function approach (Giles \& Pierce 1997) in which we consider the linearized problem with point source terms

$$
L u_{j}(x, \xi)=f_{j}(\xi) \delta(x-\xi),
$$

where $\delta(x)$ is the Dirac delta function. Using the adjoint form of the objective function (2.3), the corresponding linearized objective is

$$
I_{j}(\xi)=\int_{-1}^{1} v^{T}(x) f_{j}(\xi) \delta(x-\xi) \mathrm{d} x=v^{T}(\xi) f_{j}(\xi) .
$$

Given three linearly independent vectors $f_{j}(\xi)$, the three simultaneous equations can then be solved for the adjoint variables, giving

$$
v^{T}(\xi)=\left(I_{1}(\xi)\left|I_{2}(\xi)\right| I_{3}(\xi)\right)\left(f_{1}(\xi)\left|f_{2}(\xi)\right| f_{3}(\xi)\right)^{-1},
$$


in which the vertical lines indicate the partitioning of the row vector and array into three columns. The approach is then to choose $f_{j}(\xi)$, solve the linearized flow equations to obtain the flow perturbation $u_{j}(x, \xi)$ and the shock displacement $\delta$, evaluate $I_{j}(\xi)$ using (2.2) and, finally, obtain $v(\xi)$ from (3.2).

The key to carrying out the procedure described above is to choose a set of source vectors $f_{j}(\xi)$ which lead to relatively simple solutions to the linearized flow equations. We begin by considering isentropic flow through a converging-diverging duct with inlet, throat and outlet located at $x=-1,0,+1$, respectively. The nonlinear equations ensure that mass flux $m h \equiv \rho q h$, stagnation enthalpy $H$ and stagnation pressure $p_{0}$ all remain constant along the duct. Therefore, solutions to the linear homogeneous equations must introduce uniform perturbations to these three quantities. The general solution to the linear homogeneous equations may then be written in the form

$$
u(x)=\left.\frac{a}{h(x)} \frac{\partial U}{\partial m}(x)\right|_{H, p_{0}}+\left.b \frac{\partial U}{\partial H}(x)\right|_{p_{0}, M}+\left.c \frac{\partial U}{\partial p_{0}}(x)\right|_{H, M},
$$

where the three vectors are linearly independent and $a, b$ and $c$ represent the uniform perturbations to $m h, H$ and $p_{0}$. To simplify the analysis, perturbations to stagnation enthalpy and pressure are introduced at fixed Mach number rather than at fixed mass flux, so that non-zero values for $b$ and $c$ both imply an additional uniform perturbation to $m h$. By contrast, a non-zero value for $a$ does not perturb either $H$ or $p_{0}$.

If we now consider the inhomogeneous equations with source terms $f_{j}(\xi) \delta(x-\xi)$, the corresponding solutions

$$
u_{j}(x, \xi)=\left.a(x, \xi) \frac{1}{h(x)} \frac{\partial U}{\partial m}(x)\right|_{H, p_{0}}+\left.b(x, \xi) \frac{\partial U}{\partial H}(x)\right|_{p_{0}, M}+\left.c(x, \xi) \frac{\partial U}{\partial p_{0}}(x)\right|_{H, M}
$$

must satisfy the homogeneous equations on either side of $\xi$, and therefore $a, b, c$ will have uniform values $a_{1}, b_{1}, c_{1}$ for $x<\xi$ and $a_{2}, b_{2}, c_{2}$ for $x>\xi$. The jump conditions for the constants are obtained by integrating the dominant terms in (3.1) from $x=\xi^{-}$ to $x=\xi^{+}$, giving

$$
h(\xi)\left(\left.\left(a_{2}-a_{1}\right) \frac{1}{h(\xi)} \frac{\partial F}{\partial m}(\xi)\right|_{H, p_{0}}+\left.\left(b_{2}-b_{1}\right) \frac{\partial F}{\partial H}(\xi)\right|_{p_{0}, M}+\left.\left(c_{2}-c_{1}\right) \frac{\partial F}{\partial p_{0}}(\xi)\right|_{H, M}\right)=f_{j}(\xi) .
$$

Hence, by choosing the three linearly independent source vectors

$$
\begin{aligned}
& f_{1}(\xi)=\left.\frac{\partial F}{\partial m}(\xi)\right|_{H, p_{0}}=\left(\begin{array}{c}
1 \\
q \\
H
\end{array}\right), \\
& f_{2}(\xi)=\left.h(\xi) \frac{\partial F}{\partial H}(\xi)\right|_{p_{0}, M}=\frac{h(\xi)}{2 H}\left(\begin{array}{c}
-\rho q \\
0 \\
\rho q H
\end{array}\right), \\
& f_{3}(\xi)=\left.h(\xi) \frac{\partial F}{\partial p_{0}}(\xi)\right|_{H, M}=\frac{h(\xi)}{p_{0}}\left(\begin{array}{c}
\rho q \\
\rho q^{2}+p \\
\rho q H
\end{array}\right),
\end{aligned}
$$


the perturbations will have the simple properties

$$
\begin{aligned}
& f_{1}(\xi) \Rightarrow a_{2}-a_{1}=1, \quad b_{2}=b_{1}, \quad c_{2}=c_{1}, \\
& f_{2}(\xi) \Rightarrow b_{2}-b_{1}=1, \quad c_{2}=c_{1}, \quad a_{2}=a_{1}, \\
& f_{3}(\xi) \Rightarrow c_{2}-c_{1}=1, \quad a_{2}=a_{1}, \quad b_{2}=b_{1} .
\end{aligned}
$$

For each source vector $f_{j}(\xi)$, the three remaining unknowns in the corresponding solution $u_{j}(x, \xi)$ are determined by the three homogeneous boundary conditions appropriate to the Mach regime under consideration. These homogeneous boundary conditions are equivalent to demanding that there is no perturbation to the boundary conditions for the original nonlinear problem.

\section{Supersonic flow}

For supersonic flow, $M, H$ and $p_{0}$ are fixed at the supersonic inlet and there are no boundary conditions at the supersonic exit. Hence, for all three source vectors we require

$$
a_{1}=b_{1}=c_{1}=0
$$

to prevent perturbations to the inlet boundary conditions. Making reference to the jump relations (3.3), we then obtain

$$
\begin{array}{lll}
f_{1}(\xi) \Rightarrow a=\mathscr{H}(x-\xi), & b=0, & c=0, \\
f_{2}(\xi) \Rightarrow b=\mathscr{H}(x-\xi), & c=0, & a=0, \\
f_{3}(\xi) \Rightarrow c=\mathscr{H}(x-\xi), & a=0, & b=0,
\end{array}
$$

corresponding to the solutions

$$
\begin{aligned}
& u_{1}(x, \xi)=\left.\mathscr{H}(x-\xi) \frac{1}{h(x)} \frac{\partial U}{\partial m}(x)\right|_{H, p_{0}}, \\
& u_{2}(x, \xi)=\left.\mathscr{H}(x-\xi) \frac{\partial U}{\partial H}(x)\right|_{p_{0}, M}, \\
& u_{3}(x, \xi)=\left.\mathscr{H}(x-\xi) \frac{\partial U}{\partial p_{0}}(x)\right|_{H, M} .
\end{aligned}
$$

The objective functions are then

$$
\begin{aligned}
& I_{1}(\xi)=\left.\int_{\xi}^{1} \frac{1}{h(x)} \frac{\partial p}{\partial m}(x)\right|_{H, p_{0}} \mathrm{~d} x \\
& I_{2}(\xi)=\left.\int_{\xi}^{1} \frac{\partial p}{\partial H}(x)\right|_{p_{0}, M} \mathrm{~d} x \\
& I_{3}(\xi)=\left.\int_{\xi}^{1} \frac{\partial p}{\partial p_{0}}(x)\right|_{H, M} \mathrm{~d} x
\end{aligned}
$$

with

$$
\left.\frac{\partial p}{\partial m}(x)\right|_{H, p_{0}}=\frac{-q}{1-M^{2}},\left.\quad \frac{\partial p}{\partial H}(x)\right|_{p_{0}, M}=0,\left.\quad \frac{\partial p}{\partial p_{0}}(x)\right|_{H, M}=\frac{p}{p_{0}} .
$$


The objective function $I_{2}(\xi)$ is zero because the pressure is constant at fixed $M$ and $p_{0}$.

\section{Subsonic flow}

For subsonic flow, there are two boundary conditions on $H$ and $p_{0}$ at the subsonic inlet and one boundary condition on static pressure $p$ at the subsonic exit.

\subsection{Change in mh at fixed $H, p_{0}$}

For $f_{1}$, the inlet boundary conditions require $b=c=0$ and the exit condition requires $a_{2}=0$, corresponding to the solution and objective function

$$
u_{1}(x, \xi)=-\left.\mathscr{H}(\xi-x) \frac{1}{h(x)} \frac{\partial U}{\partial m}(x)\right|_{H, p_{0}}, \quad I_{1}(\xi)=\left.\int_{-1}^{\xi} \frac{1}{h(x)} \frac{\partial p}{\partial m}(x)\right|_{H, p_{0}} \mathrm{~d} x .
$$

\subsection{Change in $H$ at fixed $p_{0}, M$}

In this case, the inlet conditions give $b_{1}=c=0$ and the exit condition gives $a=0$, yielding a solution and objective function that are identical to the supersonic case

$$
u_{2}(x, \xi)=\left.\mathscr{H}(x-\xi) \frac{\partial U}{\partial H}(x)\right|_{p_{0}, M}, \quad I_{2}(\xi)=0 .
$$

\subsection{Change in $p_{0}$ at fixed $H, M$}

The inlet conditions now give $b=c_{1}=0$. Also, to ensure zero perturbation to the exit pressure, we require

$$
\left.\left(\left.\frac{a}{h(x)} \frac{\partial p}{\partial m}(x)\right|_{H, p_{0}}+\left.c_{2} \frac{\partial p}{\partial p_{0}}(x)\right|_{H, M}\right)\right|_{x=1}=0
$$

where $c_{2}=1$. The solution then becomes

$$
u_{3}(x, \xi)=\left.\mathscr{H}(x-\xi) \frac{\partial U}{\partial p_{0}}(x)\right|_{H, M}+\left.\frac{a}{h(x)} \frac{\partial U}{\partial m}(x)\right|_{H, p_{0}},
$$

with corresponding objective function

$$
I_{3}(\xi)=\left.\int_{\xi}^{1} \frac{\partial p}{\partial p_{0}}(x)\right|_{H, M} \mathrm{~d} x+\left.\int_{-1}^{1} \frac{a}{h(x)} \frac{\partial p}{\partial m}(x)\right|_{H, p_{0}} \mathrm{~d} x .
$$

\section{Isentropic transonic flow}

For isentropic transonic flow, $H$ and $p_{0}$ are fixed at the subsonic inlet and there are no boundary conditions at the supersonic exit. The third requirement is that the Mach number remains unity at the throat.

\subsection{Change in $m h$ at fixed $H, p_{0}$}

For $f_{1}$, the inlet boundary conditions ensure that $b=c=0$ and the throat condition requires that $a$ equals zero at the throat. Therefore, $a_{2}=0$ for $\xi<0$ and $a_{1}=0$ for 
$\xi>0$, leading to the solution

$$
u_{1}(x, \xi)=\left\{\begin{array}{cc}
-\left.\mathscr{H}(\xi-x) \frac{1}{h(x)} \frac{\partial U}{\partial m}(x)\right|_{H, p_{0}} & (\xi<0), \\
\left.\mathscr{H}(x-\xi) \frac{1}{h(x)} \frac{\partial U}{\partial m}(x)\right|_{H, p_{0}} & (\xi>0) .
\end{array}\right.
$$

Hence, if $\xi<0$, the mass flux upstream of $x=\xi$ is reduced by a unit amount, whereas if $\xi>0$, the mass flux downstream of $x=\xi$ is increased by a unit amount.

The objective function is

$$
I_{1}(\xi)=\left\{\begin{array}{cc}
-\left.\int_{-1}^{\xi} \frac{1}{h(x)} \frac{\partial p}{\partial m}(x)\right|_{H, p_{0}} \mathrm{~d} x & (\xi<0), \\
\left.\int_{\xi}^{1} \frac{1}{h(x)} \frac{\partial p}{\partial m}(x)\right|_{H, p_{0}} \mathrm{~d} x & (\xi>0) .
\end{array}\right.
$$

Since

$$
\left.\frac{\partial p}{\partial m}(x)\right|_{H, p_{0}}=\frac{-q}{1-M^{2}},
$$

and $M$ varies approximately linearly through a choked throat, then

$$
\left.\frac{\partial p}{\partial m}(x)\right|_{H, p_{0}} \sim \frac{1}{x} \quad \text { as } \quad x \rightarrow 0 .
$$

It follows that

$$
I_{1}(\xi) \sim \log (\xi) \quad \text { as } \quad \xi \rightarrow 0,
$$

so there is a logarithmic singularity in the adjoint variables at a sonic throat.

\subsection{Change in $H$ at fixed $p_{0}, M$}

In this case, the inlet conditions on $H$ and $p_{0}$ require $b_{1}=c=0$ and the throat condition gives $a=0$. The solution is then

$$
u_{2}(x, \xi)=\left.\mathscr{H}(x-\xi) \frac{\partial U}{\partial H}(x)\right|_{p_{0}, M},
$$

and the corresponding objective function, $I_{2}(\xi)$, is zero because

$$
\left.\frac{\partial p}{\partial H}(x)\right|_{p_{0}, M}=0 .
$$

\subsection{Change in $p_{0}$ at fixed $H, M$}

Now, the inlet conditions on $H$ and $p_{0}$ yield $b=c_{1}=0$, and the Mach number is fixed at the throat, so again $a=0$. The solution and linear functional thus become

$$
u_{3}(x, \xi)=\left.\mathscr{H}(x-\xi) \frac{\partial U}{\partial p_{0}}(x)\right|_{H, M}, \quad I_{3}(\xi)=\left.\int_{\xi}^{1} \frac{\partial p}{\partial p_{0}}(x)\right|_{H, M} \mathrm{~d} x .
$$

\section{Shocked flow}

For shocked flow, there are two boundary conditions on $H$ and $p_{0}$ at the subsonic inlet, the throat is again sonic, there is a shock downstream of the throat and there 
is one boundary condition on $p$ at the subsonic exit. The nonlinear equations once again ensure uniform mass flux and stagnation enthalpy throughout the duct, but the stagnation pressure now has different values on either side of the shock. Consequently, solutions to the linearized equations must now admit different but uniform stagnation pressure perturbations on either side of the shock. To account for the shock, the form of the solution must be generalized to

$$
\begin{aligned}
u_{j}\left(x, x_{s}, \xi\right)= & \left.a\left(x, x_{s}, \xi\right) \frac{1}{h(x)} \frac{\partial U}{\partial m}(x)\right|_{H, p_{0}}+\left.b\left(x, x_{s}, \xi\right) \frac{\partial U}{\partial H}(x)\right|_{p_{0}, M} \\
& +\left.c\left(x, x_{s}, \xi\right) \frac{\partial U}{\partial p_{0}}(x)\right|_{H, M}
\end{aligned}
$$

where the perturbations $a, b$, and $c$ may now be discontinuous at the shock location $x_{s}$ as well as at $\xi$.

\subsection{Shock movement}

The displacement in the shock can be calculated from the normal shock relation

$$
p_{02}=p_{01} f\left(M_{1}\right), \quad f\left(M_{1}\right)=\left(\frac{p_{2}}{p_{1}}\right)\left(\frac{1+\frac{1}{2}(\gamma-1) M_{2}^{2}}{1+\frac{1}{2}(\gamma-1) M_{1}^{2}}\right)^{\gamma / \gamma-1},
$$

with shock jump conditions

$$
\frac{p_{2}}{p_{1}}=1+\frac{2 \gamma}{\gamma+1}\left(M_{1}^{2}-1\right), \quad M_{2}^{2}=\frac{1+\left[\frac{1}{2}(\gamma-1)\right] M_{1}^{2}}{\gamma M_{1}^{2}-\frac{1}{2}(\gamma-1)},
$$

where the subscripts 1 and 2 represent quantities upstream and downstream of the shock, respectively. The perturbations to the stagnation pressure then satisfy

$$
c_{2}=c_{1} f\left(M_{1}\right)+\left.p_{01} f^{\prime}\left(M_{1}\right)\left(\frac{\mathrm{d} M}{\mathrm{~d} x} \delta+\left.\frac{a_{1}}{h(x)} \frac{\partial M}{\partial m}(x)\right|_{H, p_{0}}\right)\right|_{x=x_{s}^{-}},
$$

where $\delta$ is the resulting displacement of the shock and

$$
\left.\frac{\partial M}{\partial m}(x)\right|_{H, p_{0}}=\frac{M}{m}\left(\frac{1+\left[\frac{1}{2}(\gamma-1)\right] M^{2}}{1-M^{2}}\right) .
$$

If $h(x)$ is a piecewise differentiable function, then $\mathrm{d} M / \mathrm{d} x$ may be evaluated analytically using the area Mach number relation

$$
\left(\frac{h}{h^{*}}\right)^{2}=\frac{1}{M^{2}}\left[\frac{2}{\gamma+1}\left(1+\frac{1}{2}(\gamma-1) M^{2}\right)\right]^{(\gamma+1) /(\gamma-1)} .
$$

The throat is sonic so the sonic area $h^{*}$ is identically equal to the throat area $h_{t}$.

\subsection{Change in $m h$ at fixed $H, p_{0}$}

Since the throat is choked and $H$ and $p_{0}$ are fixed at the inlet, the form of the solution and objective function will be the same as for the isentropic transonic case when $\xi<0$. The two new scenarios to consider are when $\xi$ is between the throat and the shock, and between the shock and the exit. In either case, the mass flux perturbation will cause the shock to move and the solution must ensure that the perturbations to mass flux and stagnation enthalpy remain constant across the shock, in addition to satisfying the exit boundary condition on pressure. 
7.2.1. Perturbation between the throat and the shock $\left(0<\xi<x_{s}\right)$

The choked condition at the throat requires that all perturbations are zero for $x<\xi$. For consistency with the shock jump subscripts, perturbations between $\xi$ and the shock are denoted by $a_{1}, b_{1}, c_{1}$ and perturbations between the shock and the exit are denoted by $a_{2}, b_{2}, c_{2}$. At $\xi$, there is a unit mass flux perturbation at constant $H$ and $p_{0}$, so

$$
a_{1}=1, \quad b_{1}=0, \quad c_{1}=0 .
$$

Furthermore, $H$ remains constant for any shock location so $b_{2}=0$. The perturbation to mass flux across the shock must be constant, so

$$
a_{1}=a_{2}+\left.c_{2}\left(\left.h(x) \frac{\partial m}{\partial p_{0}}(x)\right|_{H, M}\right)\right|_{x=x_{s}^{+}} .
$$

Also, to avoid perturbing the exit pressure, we require

$$
\left.\left(\left.\frac{a_{2}}{h(x)} \frac{\partial p}{\partial m}(x)\right|_{H, p_{0}}+\left.c_{2} \frac{\partial p}{\partial p_{0}}(x)\right|_{H, M}\right)\right|_{x=1}=0 .
$$

These two equations determine the two unknowns $a_{2}$ and $c_{2}$ and equation (7.1) then determines the shock movement $\delta$. The perturbed solution is then

$u_{1}\left(x, x_{s}, \xi\right)=\left.\frac{1}{h(x)}\left[\mathscr{H}(x-\xi)+\left(a_{2}-1\right) \mathscr{H}\left(x-x_{s}\right)\right] \frac{\partial U}{\partial m}(x)\right|_{H, p_{0}}+\left.c_{2} \mathscr{H}\left(x-x_{s}\right) \frac{\partial U}{\partial p_{0}}(x)\right|_{H, M}$,

and the corresponding objective function is

$$
\begin{aligned}
I_{1}(\xi)= & \left.\int_{\xi}^{x_{s}} \frac{1}{h(x)} \frac{\partial p}{\partial m}(x)\right|_{H, p_{0}} \mathrm{~d} x \\
& +\int_{x_{s}}^{1}\left(\left.\frac{a_{2}}{h(x)} \frac{\partial p}{\partial m}(x)\right|_{H, p_{0}}+\left.c_{2} \frac{\partial p}{\partial p_{0}}(x)\right|_{H, M}\right) \mathrm{d} x-\left(p_{2}-p_{1}\right) \delta
\end{aligned}
$$

7.2.2. Perturbation between the shock and the exit $\left(x_{s}<\xi<1\right)$

All perturbations are now zero for $x<x_{s}$, so

$$
a_{1}=b_{1}=c_{1}=0,
$$

since perturbations introduced in the subsonic region following the shock cannot affect the supersonic zone. Perturbations between the shock and $\xi$ are now denoted by $a_{2}, b_{2}, c_{2}$ and perturbations between $\xi$ and the exit are denoted by $a_{3}, b_{3}, c_{3}$.

For compatibility with the upstream flow, there must be no perturbation to $H$ across the shock, so $b_{2}=b_{3}=0$. The perturbation to the stagnation pressure must be uniform throughout the subsonic region, so $c_{2}=c_{3} \equiv c$. At $\xi$, the source term produces a unit perturbation in mass flux so

$$
a_{3}-a_{2}=1 .
$$

To match the flow upstream of the shock, there must be no mass flux perturbation on the downstream side of the shock

$$
a_{2}+\left.c\left(\left.h(x) \frac{\partial m}{\partial p_{0}}(x)\right|_{H, M}\right)\right|_{x=x_{s}^{+}}=0 .
$$


Also, to ensure zero perturbation of the exit static pressure we require,

$$
\left.\left(\left.\frac{a_{3}}{h(x)} \frac{\partial p}{\partial m}(x)\right|_{H, p_{0}}+\left.c \frac{\partial p}{\partial p_{0}}(x)\right|_{H, M}\right)\right|_{x=1}=0,
$$

giving three equations for the three unknowns. The perturbed solution then has the form

$$
u_{1}\left(x, x_{s}, \xi\right)=\left.\frac{1}{h(x)}\left[a_{2} \mathscr{H}\left(x-x_{s}\right)+\mathscr{H}(x-\xi)\right] \frac{\partial U}{\partial m}(x)\right|_{H, p_{0}}+\left.c \mathscr{H}\left(x-x_{s}\right) \frac{\partial U}{\partial p_{0}}(x)\right|_{H, M},
$$

with objective function

$$
\begin{aligned}
I_{1}(\xi)= & \left.\int_{x_{s}}^{\xi} \frac{a_{2}}{h(x)} \frac{\partial p}{\partial m}(x)\right|_{H, p_{0}} \mathrm{~d} x+\left.\int_{\xi}^{1} \frac{a_{3}}{h(x)} \frac{\partial p}{\partial m}(x)\right|_{H, p_{0}} \mathrm{~d} x \\
& +\left.\int_{x_{s}}^{1} c \frac{\partial p}{\partial p_{0}}(x)\right|_{H, M} \mathrm{~d} x-\left(p_{2}-p_{1}\right) \delta .
\end{aligned}
$$

\subsection{Change in $H$ at fixed $p_{0}, M$}

Ahead of the shock, the perturbation to stagnation pressure $c$ must be zero owing to the inlet boundary condition, and the mass flux perturbation $a$ must be zero owing to the choked throat. The inlet condition on $H$ ensures the perturbation to stagnation enthalpy is zero for $x<\xi$, and the unit jump in $b$ at $\xi$ will produce a uniform perturbation in $H$ across the shock, without affecting the exit condition on pressure.

There still exists the possibility that $a$ and $c$ are non-zero constants following the shock, balancing to produce zero mass flux perturbation at the shock

$$
\left.\left(a+\left.\operatorname{ch}(x) \frac{\partial m}{\partial p_{0}}(x)\right|_{H, M}\right)\right|_{x=x_{s}^{+}}=0,
$$

and zero pressure perturbation at the exit

$$
\left.\left(\left.\frac{a}{h(x)} \frac{\partial p}{\partial m}(x)\right|_{H, p_{0}}+\left.c \frac{\partial p}{\partial p_{0}}(x)\right|_{H, M}\right)\right|_{x=1}=0 .
$$

However, the determinant of this system is non-zero, so there is only the trivial solution $a=c=0$. Hence, the solution and objective function in the shocked case have the form

$$
u_{2}\left(x, x_{s}, \xi\right)=\left.\mathscr{H}(x-\xi) \frac{\partial U}{\partial H}(x)\right|_{p_{0}, M}, \quad I_{2}(\xi)=0,
$$

and there is no displacement of the shock.

\subsection{Change in $p_{0}$ at fixed $H, M$}

For shocked flow with a unit jump in stagnation pressure, the presence of the shock affects the perturbed solution for all locations of $\xi$. This is in contrast to the shocked case with a jump in mass flux, where the solution remained unchanged from the isentropic transonic case for $\xi<0$. The two scenarios to consider in the present case are when $\xi$ is between the inlet and the shock, and between the shock and the exit. 
7.4.1. Perturbation between the inlet and the shock $\left(-1<\xi<x_{s}\right)$

As in the shock-free case, there is no perturbation for $x<\xi$. Denoting the perturbations between $\xi$ and the shock by $a_{1}, b_{1}, c_{1}$ and those after the shock by $a_{2}, b_{2}, c_{2}$, we have by definition

$$
a_{1}=0, \quad b_{1}=0, \quad c_{1}=1 .
$$

The perturbation to $H$ must be constant across the shock so $b_{2}=0$. Constant mass flux perturbation at the shock requires

$$
\left.c_{1}\left(\left.h(x) \frac{\partial m}{\partial p_{0}}(x)\right|_{H, M}\right)\right|_{x=x_{s}^{-}}=a_{2}+\left.c_{2}\left(\left.h(x) \frac{\partial m}{\partial p_{0}}(x)\right|_{H, M}\right)\right|_{x=x_{s}^{+}},
$$

and zero perturbation to the exit pressure is ensured by setting

$$
\left.\left(\left.\frac{a_{2}}{h(x)} \frac{\partial p}{\partial m}(x)\right|_{H, p_{0}}+\left.c_{2} \frac{\partial p}{\partial p_{0}}(x)\right|_{H, M}\right)\right|_{x=1}=0,
$$

providing two equations for the two unknowns. The solution then has the form

$u_{3}\left(x, x_{s}, \xi\right)=\left.\left[\mathscr{H}(x-\xi)+\left(c_{2}-1\right) \mathscr{H}\left(x-x_{s}\right)\right] \frac{\partial U}{\partial p_{0}}(x)\right|_{H, M}+\left.\frac{a_{2}}{h(x)} \mathscr{H}\left(x-x_{s}\right) \frac{\partial U}{\partial m}(x)\right|_{H, p_{0}}$,

with corresponding objective function

$$
\begin{aligned}
I_{3}\left(x, x_{s}, \xi\right)= & \left.\int_{\xi}^{x_{s}} \frac{\partial p}{\partial p_{0}}(x)\right|_{H, M} \mathrm{~d} x \\
& +\int_{x_{s}}^{1}\left(\left.\frac{a_{2}}{h(x)} \frac{\partial p}{\partial m}(x)\right|_{H, p_{0}}+\left.c_{2} \frac{\partial p}{\partial p_{0}}(x)\right|_{H, M}\right) \mathrm{d} x-\left(p_{2}-p_{1}\right) \delta .
\end{aligned}
$$

7.4.2. Perturbation between the shock and the exit $\left(x_{s}<\xi<1\right)$

There are now no perturbations upstream of the shock, so

$$
a_{1}=b_{1}=c_{1}=0 .
$$

Perturbations in the region between the shock and $\xi$ are denoted by $a_{2}, b_{2}, c_{2}$ and those between $\xi$ and the exit are denoted by $a_{3}, b_{3}, c_{3}$.

Compatibility at the shock and the fact that $m h$ and $p_{0}$ are perturbed at constant $H$, together imply that there are no perturbations to stagnation enthalpy following the shock, so $b_{2}=b_{3}=0$. Perturbations to the mass flux must be constant throughout the subsonic region $\left(a_{2}=a_{3} \equiv a\right)$ since the jump condition at $\xi$ corresponds solely to a unit perturbation in stagnation pressure

$$
c_{3}-c_{2}=1 \text {. }
$$

Zero mass flux perturbation at the shock then gives

$$
a+\left.c_{2}\left(\left.\frac{\partial m}{\partial p_{0}}(x)\right|_{H, M}\right)\right|_{x=x_{s}^{+}}=0
$$

and zero perturbation to the exit pressure requires

$$
\left.\left(\left.\frac{a}{h(x)} \frac{\partial p}{\partial m}(x)\right|_{H, p_{0}}+\left.c_{3} \frac{\partial p}{\partial p_{0}}(x)\right|_{H, M}\right)\right|_{x=1}=0,
$$



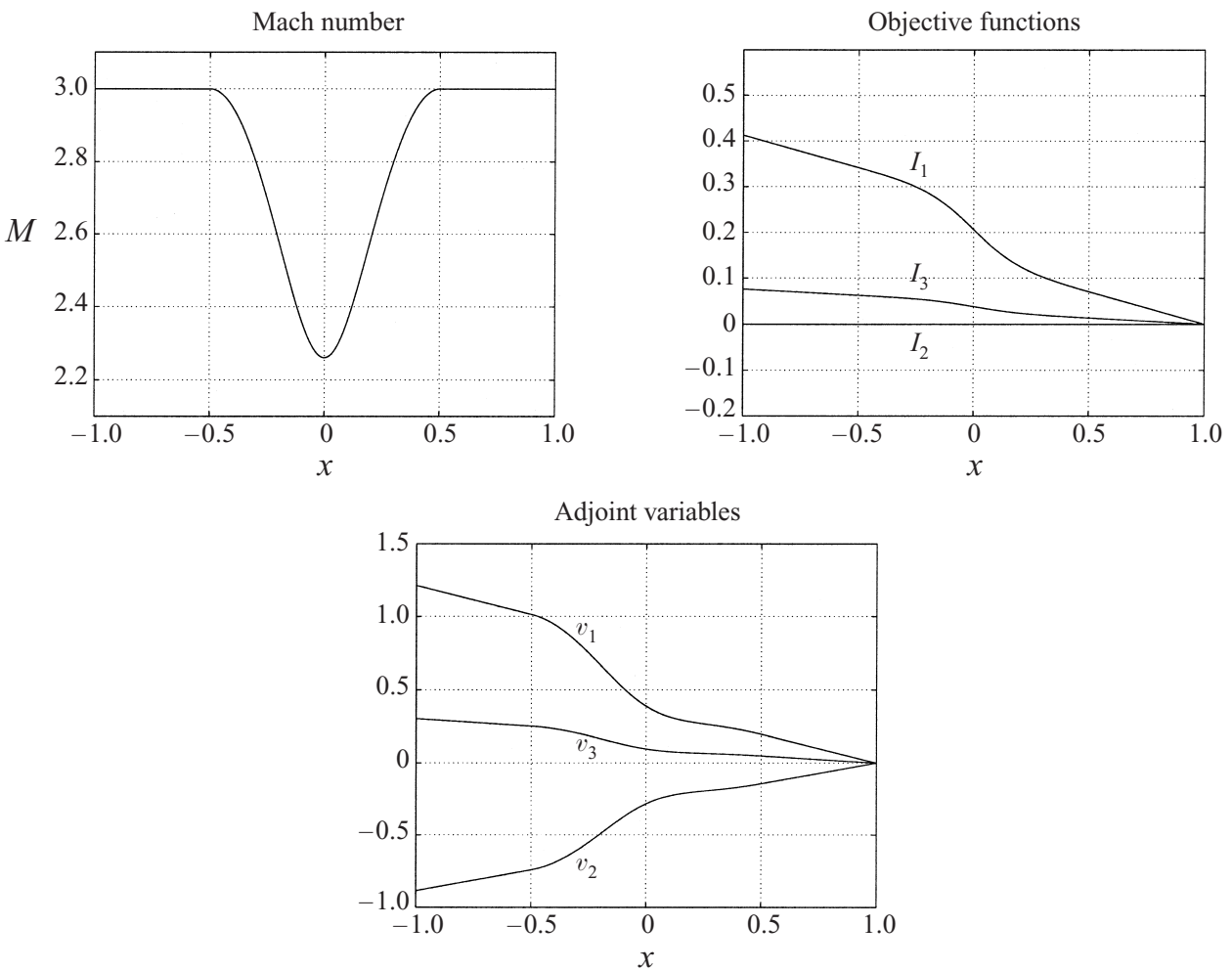

FIGURE 1. Mach number, objective functions and adjoint variables for supersonic flow conditions.

$$
M_{\text {in }}=3, H_{\text {in }}=4, p_{0 \text { in }}=2 \text {. }
$$

providing three equations for three unknowns. The solution has the form

$$
u_{3}\left(x, x_{s}, \xi\right)=\left.\left[c_{2} \mathscr{H}\left(x-x_{s}\right)+\mathscr{H}(x-\xi)\right] \frac{\partial U}{\partial p_{0}}(x)\right|_{H, M}+\left.\frac{a}{h(x)} \mathscr{H}\left(x-x_{s}\right) \frac{\partial U}{\partial m}(x)\right|_{H, p_{0}},
$$

with corresponding objective function

$$
\begin{aligned}
I_{3}(\xi)= & \left.\int_{x_{s}}^{1} \frac{a}{h(x)} \frac{\partial p}{\partial m}(x)\right|_{H, p_{0}} \mathrm{~d} x+\left.\int_{x_{s}}^{\xi} c_{2} \frac{\partial p}{\partial p_{0}}(x)\right|_{H, M} \mathrm{~d} x \\
& +\left.\int_{\xi}^{1} c_{3} \frac{\partial p}{\partial p_{0}}(x)\right|_{H, M} \mathrm{~d} x-\left(p_{2}-p_{1}\right) \delta .
\end{aligned}
$$

\section{Sample solutions}

The analytic objective functions $I(\xi)$ and adjoint solutions $v(\xi)$ corresponding to supersonic, subsonic, isentropic and shocked transonic flows are shown in figures 1 to 4. The boundary conditions for these test cases are defined in the figure captionsand the geometric definition of the duct is given by

$$
h(x)= \begin{cases}2 & \left(-1 \leqslant x \leqslant-\frac{1}{2}\right), \\ 1+\sin ^{2}(\pi x) & \left(-\frac{1}{2}<x<\frac{1}{2}\right), \\ 2 & \left(\frac{1}{2} \leqslant x \leqslant 1\right) .\end{cases}
$$



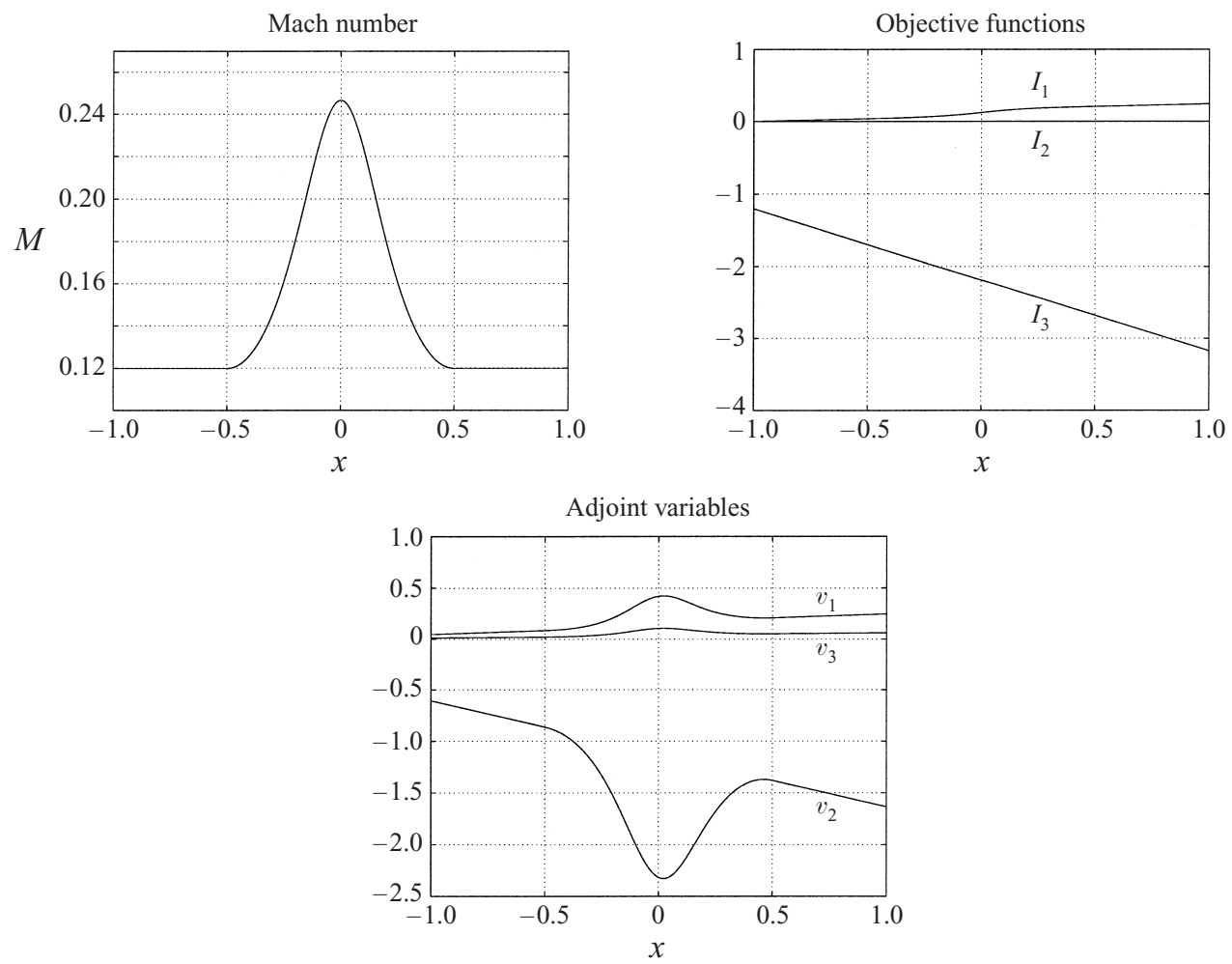

FIGURE 2. Mach number, objective functions and adjoint variables for subsonic flow conditions. $H_{\text {in }}=4, p_{0_{\text {in }}}=2, p_{\text {ex }}=1.98$.

The analytic results have been verified using numerical solutions obtained by discretizing the adjoint equation (2.4) directly (Giles \& Pierce 1998). For the supersonic case of figure 1, the adjoint variables are all zero at the exit, as required to eliminate the dependence on $u$ of the boundary term (2.6) in the adjoint derivation. For the isentropic transonic case of figure 3, the logarithmic singularity in $I_{1}$ at the sonic throat is reflected in the singularities of all three adjoint variables. For the shocked case of figure 4, the objective functions are discontinuous at the shock, but the adjoint variables are continuous with zero gradient, as proved earlier.

\section{Conclusions}

In this paper, we have undertaken a detailed investigation of adjoint solutions for the quasi-one-dimensional Euler equations, focusing in particular on the solution behaviour at a shock or a sonic point where there is a change in sign of one of the hyperbolic characteristics.

Formulating the adjoint equations using Lagrange multipliers to enforce the Rankine-Hugoniot shock jump conditions proves that, contrary to previous literature, the adjoint variables are continuous at the shock. This result is supported by the derivation of a closed form solution to the adjoint equations using a Green's function approach. In addition to proving the existence of a $\log (x)$ singularity at the sonic point, this closed form solution should be very helpful as a test case for others developing numerical methods for the adjoint equations. 

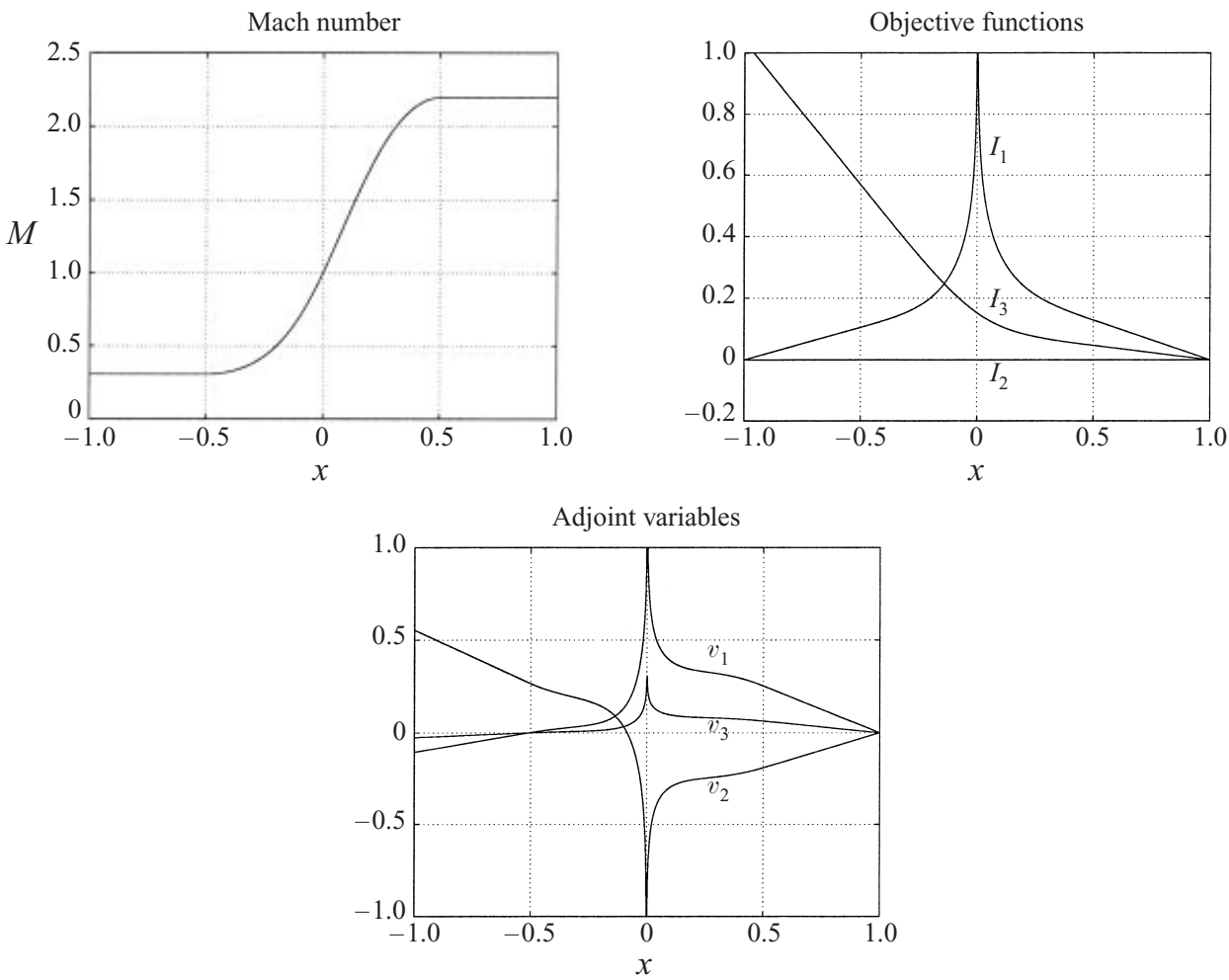

FIGURE 3. Mach number, objective functions and adjoint variables for isentropic transonic flow conditions. $H_{\text {in }}=4, p_{0 \text { in }}=2$.

Future research will attempt to extend this analysis to two dimensions. Preliminary analysis, supported by the results of numerical computations (Giles \& Pierce 1997), shows that the adjoint variables are again continuous at a shock, and that an adjoint boundary condition is required along the length of the shock. However, since adjoint computations currently employed for transonic aerofoil optimization do not enforce this internal boundary condition, it remains an open question as to whether there is a consistency error in the limit of increasing grid resolution. In two dimensions, numerical evidence suggests that there is no longer a singularity at a sonic line if (as is usually the case) it is not orthogonal to the flow. This can be explained qualitatively by considering the region of influence of points in the neighbourhood of the sonic line (Giles \& Pierce 1997). An important new feature that must be considered for two-dimensional flows is the behaviour of the adjoint solution at stagnation points. Here, the analysis indicates an inverse square-root singularity along the incoming stagnation streamline, but further numerical experiments are required to confirm this behaviour.

An improved understanding of the behaviour of adjoint solutions is necessary both to rigorously establish the theoretical basis for engineering optimal design methods and to illuminate the role of the adjoint solution in numerical error analysis. In this latter setting, the adjoint solution reveals the sensitivity of a functional, such as lift, to the truncation errors associated with the numerical discretization. Where there are singularities in the adjoint variables it is desirable to greatly increase the grid resolution so as to reduce the contribution of the local truncation error to the 

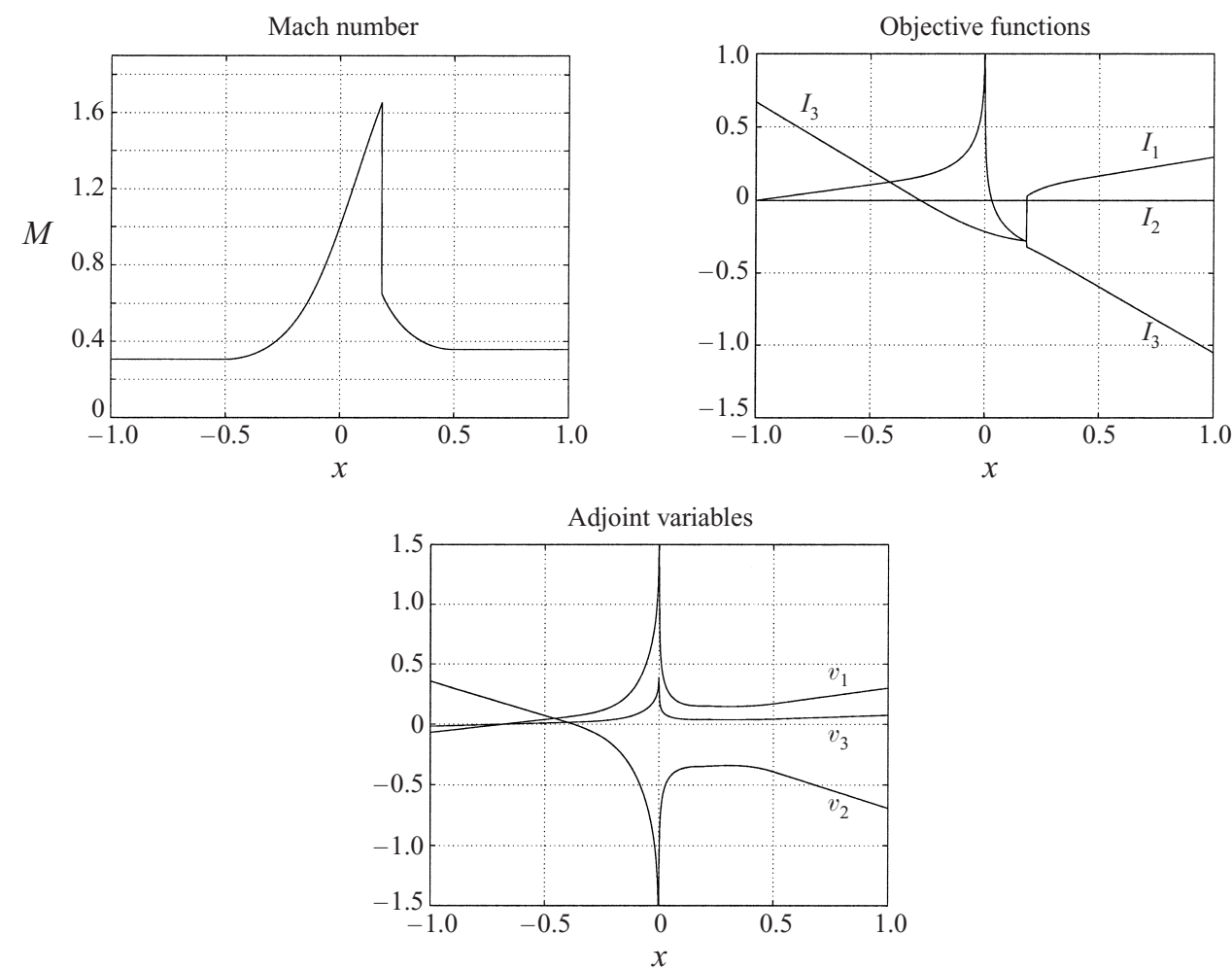

FigURE 4. Mach number, objective functions and adjoint variables for shocked flow conditions.

$$
H_{\text {in }}=4, p_{0 \text { in }}=2, p_{\text {ex }}=1.6 \text {. }
$$

error in the functional. Thus, adjoint analysis offers a rigorous basis for optimal grid adaptation (Venditti \& Darmofal 1999). Furthermore, by estimating the truncation error in the original nonlinear numerical solution, and using the adjoint solution to estimate the consequential error in the functional of interest, an improved estimate can be obtained with twice the order of accuracy (Giles \& Pierce 1998, 1999; Pierce \& Giles 1998, 2000). Future developments along these lines will lead to great improvements in accuracy for key engineering quantities such as lift and drag.

This research was supported by EPSRC under grant GR/K91149.

\section{REFERENCES}

Anderson, W. \& Bonhaus, D. 1999 Airfoil design on unstructured grids for turbulent flows. AIAA J. 37, 185-191.

BECKER, R. \& RANNACHER, R. 1998 Weighted a posteriori error control in finite element methods. In Proceedings of ENUMATH-97 (ed. H. G. Block et al.), pp. 621-637. World Scientific Publishing.

Cliff, E., Heinkenschloss, M. \& Shenoy, A. 1996 On the optimality system for a 1-D Euler flow problem. AIAA Paper 96-3993-CP.

Cliff, E., Heinkenschloss, M. \& Shenoy, A. 1998 Adjoint-based methods for aerodynamic design optimisation. In Computational Methods for Optimal Design (ed. J. Borggaard, J. Burns, E. Cliff \& S. Schreck). Birkhäuser.

Elliott, J. \& Peraire, J. 1997 Practical 3D aerodynamic design and optimization using unstructured meshes. AIAA J. 35, 1479-1485. 
Giles, M. \& Pierce, N. 1997 Adjoint equations in CFD: duality, boundary conditions and solution behaviour. AIAA Paper 97-1850.

Giles, M. \& Pierce, N. 1998 On the properties of solutions of the adjoint Euler equations. In Numerical Methods for Fluid Dynamics VI (ed. M. Baines). ICFD.

Giles, M. \& Pierce, N. 1999 Improved lift and drag estimates using adjoint Euler equations. AIAA Paper 99-3293.

Giles, M. \& Pierce, N. 2001 An introduction to the adjoint approach to design. Flow, Turbulence Combust. (to appear).

Iollo, A. \& Salas, M. 1996 Contribution to the optimal shape design of two-dimensional, internal flows with embedded shocks. J. Comput. Phys. 125, 124-134.

Iollo, A., Salas, M. \& TA'ASAN, S. 1993 Shape optimization governed by the Euler equations using an adjoint method. ICASE Report 93-78.

JAmeson, A. 1988 Aerodynamic design via control theory. J. Sci. Comput. 3, 233-260.

JAMESON, A. 1995 Optimum aerodynamic design using control theory. In Computational Fluid Dynamics review 1995 (ed. M. Hafez \& K. Oshima), pp. 495-528. John Wiley.

JAMESON, A. 1999 Re-engineering the design process through computation. J. Aircraft 36, 36-50.

Jameson, A., Pierce, N. \& Martinelli, L. 1998 Optimum aerodynamic design using the NavierStokes equations. J. Theor. Comput. Fluid Mech. 10, 213-237.

Johnson, C., RannaCher, R. \& Boman, M. 1995 Numerics and hydrodynamic stability-toward error control in computational fluid dynamics. SIAM J. Numer. Anal. 32, 1058-1079.

Newman, J., Taylor, A., Barnwell, R., Newman, P. \& Hou, G. J.-W. 1999 Overview of sensitivity analysis and shape optimization for complex aerodynamic configurations. J. Aircraft 36, 87-96.

Paraschivoiu, M., Peraire, J. \& Patera, A. 1997 A posteriori finite element bounds for linearfunctional outputs of elliptic partial differential equations. Comput. Meth. Appl. Mech. Engng 150, 289-312.

PierCe, N. \& GiLES, M. 1998 Adjoint recovery of superconvergent functionals from approximate solutions of partial differential equations. Tech. Rep. NA98/18. Oxford University Computing Laboratory.

PierCe, N. \& Giles, M. 2000 Adjoint recovery of superconvergent functionals from PDE approximations. SIAM Rev. 42, 247-264.

Reuther, J., Jameson, A., Alonso, J., Remlinger, M. \& Saunders, D. $1999 a$ Constrained multipoint aerodynamic shape optimisation using an adjoint formulation and parallel computers, part 1 . J. Aircraft 36, 51-60.

Reuther, J., Jameson, A., Alonso, J., Remlinger, M. \& Saunders, D. $1999 b$ Constrained multipoint aerodynamic shape optimisation using an adjoint formulation and parallel computers, part 2. J. Aircraft 36, 61-74.

Reuther, J., Jameson, A., Farmer, J., Martinelli, L. \& Saunders, D. 1996 Aerodynamic shape optimization of computer aircraft configurations via an adjoint formulation. AIAA Paper 96-0094.

SüLI, E. 1998 A posteriori error analysis and adaptivity for finite element approximations of hyperbolic problems. In An Introduction to Recent Developments in Theory and Numerics for Conservation Laws (ed. M. O. D. Kröner \& C. Rohde). Lecture Notes in Computational Science and Engineering, vol. 5, pp. 123-194. Springer.

Venditti, D. \& Darmofal, D. 1999 A multilevel error estimation and grid adaptive strategy for improving the accuracy of integral outputs. AIAA Paper 99-3292. 